\title{
Evaluación in Vitro de la Biocompatibilidad de Cuatro Cementos Selladores con Osteoblastos Humanos
}

\author{
In vitro Evaluation of Four Sealer Cements Biocompatibility with Human Osteoblasts
}

\author{
Ramos-López, M. ${ }^{1}$; Silva-Benítez, E. L.'; Aguilar-Medina, M.2; Ayala-Ham, A. R.1; Romero-Quintana, J. \\ G. ${ }^{2}$; Soto-Sainz, J. E. ${ }^{1}$; Velázquez-Solano, A. ${ }^{1}$; Ramos-Payan, R. ${ }^{2}$ \& Castro-Salazar, G. Y. ${ }^{1}$
}

RAMOS-LÓPEZ, M.; SILVA-BENÍTEZ, E. L.; AGUILAR-MEDINA, M.; AYALA-HAM, A. R.; ROMERO-QUINTANA, J. G.; SOTO-SAINZ, J. E.; VELÁZQUEZ-SOLANO, A.; RAMOS-PAYAN, R. \& CASTRO-SALAZAR, G. Y. Evaluación in vitro de la biocompatibilidad de cuatro cementos selladores con osteoblastos humanos. Int. J. Odontostomat., 13(1):64-68, 2019.

RESUMEN: El correcto sellado apical es un paso importante durante el tratamiento de conductos, para esto, se utilizan puntas de gutapercha y cemento sellador, de este último existen diversas formulaciones químicas en el mercado, por lo cual es importante tomar en cuenta los efectos que estas pueden tener en el proceso de cicatrización periapical. El propósito de este estudio fue evaluar la biocompatibilidad de cuatro cementos selladores con diferente composición química con osteoblastos humanos. Se prepararon extractos de cementos selladores a con dos concentraciones (10 mg/mL y 40 $\mathrm{mg} / \mathrm{mL}$ ) y dos tiempos de exposición (10 min y $8 \mathrm{~h}$ ), estos fueron colocados en contacto con osteoblastos humanos para evaluar la proliferación y citotoxicidad a 24, 72 y $96 \mathrm{~h}$ con sus respectivos controles y blancos. Se realizó un análisis estadístico con ANOVA de un factor y la prueba de comparaciones múltiple de Bonferroni. Los resultados obtenidos, tanto en el ensayo de citotoxicidad como en el de proliferación, indicaron que el cemento a base de resina no es biocompatible con osteoblastos. El cemento a base de poli-dimetilxilosano fue el único que no mostró citotoxicidad a ningún de tiempo de exposición y concentración examinadas en este estudio.

PALABRAS CLAVE: biocompatibilidad, cementos selladores, osteoblastos.

\section{INTRODUCCIÓN}

El tratamiento de conductos tiene como finalidad eliminar la infección y sellar completamente el canal radicular para evitar la filtración de líquidos o bacterias (Eldeniz et al., 2007), evitando la reinfección de la zona y el posible fracaso del tratamiento, ya que la infección bacteriana presente en pulpa dental puede llevar a lesiones periapicales (Fernandes \& de Ataide, 2010). La función de los cementos selladores endodónticos es cerrar los espacios que podrían quedar en el canal, sin ser extruidos hacia los tejidos perirradiculares (Shon et al., 2012). Aún con los cuidados necesarios, existen situaciones en las que los selladores sobrepasan el canal radicular incrementando el riesgo de irritación del tejido o retraso en la cicatrización (Brackett et al., 2010), el cual puede ser mayor si no son altamente biocompatibles.

Existen diferentes tipos de selladores de acuerdo a su composición química (Scelza et al., 2012), y respecto a esta característica han sido divididos en 5 grupos (Garrido et al., 2015): selladores a base de hidróxido de calcio, óxido de zinc eugenol, resina, ionómero de vidrio y silicona; al colocar estos materiales se encontrarán en contacto con tejidos periapicales por tiempos prolongados, por lo que existe la posibilidad de que productos de degradación sean liberados a través de los túbulos dentinarios o canales laterales y accesorios, pudiendo dañar el ligamento periodontal y el hueso alveolar (Bernáth \& Szabó, 2003) y afectar las actividades metabólicas normales del hueso, lo cual podría poner en riesgo la remodelación y regeneración ósea (Rodrigues et al., 2014). La última meta de la terapia endodóntica debe ser regresar al órgano dentario tratado a un estado de salud y funcionalidad sin la necesidad de realizar ningún tipo de intervención quirúrgica (Fernandes \& de Ataide). Debido a esto, es importante evaluar la biocompatibilidad de los cementos selladores con osteoblastos, ya algunos estu-

${ }^{1}$ Facultad de Odontología, Universidad Autónoma de Sinaloa, México.

${ }^{2}$ Facultad de Ciencias Químico Biológicas, Universidad Autónoma de Sinaloa, México. 
RAMOS-LÓPEZ, M.; SILVA-BENÍTEZ, E. L.; AGUILAR-MEDINA, M.; AYALA-HAM, A. R.; ROMERO-QUINTANA, J. G.; SOTO-SAINZ, J. E.; VELÁZQUEZ-SOLANO, A.; RAMOSPAYAN, R. \& CASTRO-SALAZAR, G. Y. Evaluación in vitro de la Biocompatibilidad de Cuatro Cementos Selladores con Osteoblastos Humanos. Int. J. Odontostomat., 13(1):64-68, 2019.

dios sugieren que son capaces de interferir en la producción de moléculas como COX-2 (Huang et al., 2003) y RANKL (Huang et al., 2009; Kim et al., 2013a,b), esta última íntimamente relacionada con la diferenciación de células óseas y osteoclastos para formar tejido óseo (Gerstenfeld et al., 2003). Además de que la presencia de cemento sellador en el periápice ha sido relacionada como una de las causas de periodontitis apical persistente (Nair, 2006).

El propósito de este estudio fue evaluar la biocompatibilidad in vitro, mediante ensayos de proliferación y citotoxicidad, con una línea celular primaria humana de osteoblastos, al ser colocada en contacto con cuatro selladores de conductos con diferente composición química: óxido de zinc eugenol (Sealapex; SybroEndo, CA, USA), resina epóxica (AH Plus; Dentsply, Konstanz, Alemania), silicato de calcio (MTA Fillapex; Angelus, PR, Brasil) y poli-dimetilxilosano (Roeko Seal; Coltene/Whaledent, Langenau, Alemania) a diferentes concentraciones y tiempos de exposición. Es importante señalar que hasta el momento son muy pocos los estudios que han evaluado los efectos citotóxicos de MTA Fillapex (Salles et al., 2012; Scelza et al.), Sealapex (Al-Awadhi et al., 2004; Lee et al., 2007; Scelza et al.), AH Plus (Pinna et al., 2008; $\mathrm{Xu}$ et al., 2010; Loushine et al., 2011; Ehsani et al., 2012; Kim et al., 2013a,b; Garrido et al.) y Roeko Seal (Al-Awadhi et al.) en osteoblastos, además de que la mayoría de estos son en células de otra especie.

\section{MATERIAL Y MÉTODO}

Preparación de los selladores y extractos de medio de cultivo. Bajo condiciones de esterilidad se preparó cada cemento sellador de acuerdo a las instrucciones del fabricante. Las muestras preparadas de cada cemento fueron colocadas en medio de cultivo, el cual consistió en Medio Eagle Modificado por Dulbecco (DMEM, Sigma-Aldrich) suplementado con antibiótico al $1 \%$ (Penstrep, Gibco) para lograr una concentración de 10 o $40 \mathrm{mg} / \mathrm{ml}$, además se agregaron perlas de vidrio de $0,5 \mathrm{~mm}$ de diámetro para homogenizar la solución durante un minuto con ayuda de un vórtex (Maxi Mix II, Barnstead International).

Se prepararon cuatro tipos de extractos según el tiempo de liberación que tuvieron con los diferentes cementos y su concentración. Los extractos a dos concentraciones (10 y $40 \mathrm{mg} / \mathrm{ml}$ ) se incubaron por $10 \mathrm{~min}$ (tiempo de extracción 1) u 8 h (tiempo de extracción
2) a $37{ }^{\circ} \mathrm{C}$ a $250 \mathrm{rpm}$ (Enviroment Shaker, Lab-Line Instruments), posteriormente se centrifugaron (5417R, Eppendorf) a 13000 rpm durante 5 min y se recuperó el sobrenadante, el cual fue conservado a $4{ }^{\circ} \mathrm{C}$ hasta la realización de los experimentos.

Un control de medio (DMEM suplementado con antibiótico) fue tratado bajo las mismas condiciones que los extractos.

Exposición de las líneas celulares a los extractos. La línea celular de cultivo primario (osteoblastos) fue donada por el Laboratorio de Ciencia Básica de la Facultad de Estomatología de la Universidad Autónoma de San Luis Potosí. Al presentar un $90 \%$ de confluencia estas células fueron despegadas con Tripsina/EDTA al $0,25 \%$ (Gibco) y centrifugadas a 1,000 rpm durante 10 min. La suspensión celular fue resuspendida en 1 $\mathrm{ml}$ de medio para realizar el conteo celular mediante azul de tripano con ayuda de una cámara de Neubauer.

Los extractos preparados fueron atemperados y se colocaron $100 \mu$ l en placas de 96 pozos (Thermo Scientific) por triplicado con 10 mil células, a todos los pozos se les agregó $100 \mu \mathrm{l}$ de DMEM suplementado con suero bovino fetal (Gibco) al $10 \%$ y antibiótico al $1 \%$. Para los ensayos se utilizaron los controles y blancos correspondientes, todos los ensayos fueron realizados a 24,72 y $96 \mathrm{~h}$.

Análisis citotoxicidad. Se utilizó el kit comercial CytoTox 96 Non-Radioactive Cytotoxicity Assay (Promega) siguiendo las instrucciones del fabricante. Para este análisis se utilizó un control de citotoxicidad máxima y un control de muerte normal. Las lecturas de absorbancia fueron tomadas a $490 \mathrm{~nm}$.

Análisis de proliferación. Se utilizó el kit comercial CellTiter 96 Non-Radioactive Cell Proliferation Assay (Promega) siguiendo las instrucciones del fabricante, posterior a los tiempos de incubación (24, 72 y 96 h) se agregaron $15 \mu \mathrm{L}$ la solución de tinción para realizar una incubación a $37^{\circ} \mathrm{C}$ por $3 \mathrm{~h}$. Transcurrido el tiempo se adicionaron $100 \mu \mathrm{L}$ de la solución de paro y se colocó a $4{ }^{\circ} \mathrm{C}$ durante toda la noche. Al siguiente día se tomó lectura en un lector de microplacas (ELIREAD, KontroLab) a $570 \mathrm{~nm}$.

Análisis estadístico. Se realizó Análisis de Varianza (ANOVA) de un factor y la prueba de comparaciones múltiple de Bonferroni. La significancia estadística se marcó con un valor de $p<0.05$ Todos los datos se analizaron con el programa IBM SPSS v20 (IL, USA). 


\section{RESULTADOS}

Análisis de citotoxicidad. El cemento a base de resina epóxica fue el que mostró en general una citotoxicidad mayor en comparación tanto con el promedio de muerte normal como con los demás cementos en todos los grupos evaluados (Tablas I y II), incluso presenta en ambos tiempos de extracción, en todas las concentraciones y horas de exposición diferencia estadísticamente significativa con la citotoxicidad normal.

Después de este material, el cemento a base de óxido de zinc eugenol muestra una mayor citotoxicidad en comparación con el control de citotoxicidad normal.
El cemento que no presentó citotoxicidad es el fabricado a base de poli-dimetilxilosano mostrando en ambos tiempos de extracción y en todas las concentraciones y horas de evaluación una citotoxicidad similar al control de muerte normal.

Análisis de proliferación. Todos los grupos que fueron expuestos al material a base de resina epóxica a los diferentes tiempos de extracción y concentraciones evaluadas (Tablas III y IV) mostraron una proliferación muy por debajo de la normal, mostrando en todos los casos una diferencia estadísticamente significativa en relación al grupo de proliferación normal, lo que concuerda con los resultados obtenidos en el análisis de citotoxicidad.

Tabla I. Resultados de absorbancia obtenidos del análisis de citotoxicidad en osteoblastos exposición al material extraído durante 10 minutos.

\begin{tabular}{lllllll}
\multicolumn{2}{c}{ Grupos } & \multicolumn{2}{c}{$24 \mathrm{~h}$} & \multicolumn{2}{c}{$72 \mathrm{~h}$} & \multicolumn{2}{c}{$96 \mathrm{~h}$} \\
& $10 \mathrm{mg} / \mathrm{ml}$ & $40 \mathrm{mg} / \mathrm{ml}$ & $10 \mathrm{mg} / \mathrm{ml}$ & $40 \mathrm{mg} / \mathrm{ml}$ & $10 \mathrm{mg} / \mathrm{ml}$ & $40 \mathrm{mg} / \mathrm{ml}$ \\
\hline SC & $0,14 \pm 0,04^{\mathrm{a}}$ & $0,15 \pm 0,03^{\mathrm{a}}$ & $0,23 \pm 0,03 \mathrm{a}$ & $0,5 \pm 0,1^{\mathrm{a}, \mathrm{c}, \mathrm{d}}$ & $0,26 \pm 0,05^{\mathrm{a}}$ & $0,51 \pm 0,01^{\mathrm{a}}$ \\
$\mathrm{RE}$ & $0,60 \pm 0,09^{\mathrm{b}}$ & $0,67 \pm 0,07^{\mathrm{b}}$ & $0,69 \pm 0,07 \mathrm{~b}$ & $0,76 \pm 0,03^{\mathrm{a}, \mathrm{b}}$ & $0,95 \pm 0,23^{\mathrm{b}}$ & $0,92 \pm 0,3^{\mathrm{b}}$ \\
PDM & $0,21 \pm 0,04^{\mathrm{a}}$ & $0,15 \pm 0,05^{\mathrm{a}}$ & $0,18 \pm 0,01 \mathrm{a}$ & $0,2 \pm 0,03^{\mathrm{c}}$ & $0,25 \pm 0,01^{\mathrm{a}}$ & $0,26 \pm 0,01^{\mathrm{a}}$ \\
OZE & $0,21 \pm 0,02^{\mathrm{a}}$ & $0,59 \pm 0^{\mathrm{b}}$ & $0,45 \pm 0,05 \mathrm{c}$ & $0,73 \pm 0,29^{\mathrm{b}, \mathrm{d}}$ & $0,45 \pm 0,03^{\mathrm{a}}$ & $0,66 \pm 0,08^{\mathrm{a}, \mathrm{b}}$ \\
Control & $0,26 \pm 0,03^{\mathrm{a}}$ & $0,26 \pm 0,03^{\mathrm{a}}$ & $0,2 \pm 0,03^{\mathrm{a}}$ & $0,2 \pm 0,03^{\mathrm{c}, \mathrm{e}}$ & $0,26 \pm 0,01^{\mathrm{a}}$ & $0,26 \pm 0,01^{\mathrm{a}}$ \\
\hline
\end{tabular}

SC: silicato de calcio, RE: resina epóxica, PDM: poli-dimetilsiloxano, OZE: óxido de zinc eugenol, Control: citotoxicidad normal. Los valores de la misma columna que no comparten el mismo superíndice son significativamente diferentes en $p<.05$.

Tabla II. Resultados de absorbancia obtenidos del análisis de citotoxicidad en osteoblastos exposición al material extraído durante 8 horas.

\begin{tabular}{lllllll}
\hline \multirow{2}{*}{ Grupos } & \multicolumn{2}{c}{$24 \mathrm{~h}$} & \multicolumn{2}{c}{$72 \mathrm{~h}$} & \multicolumn{2}{c}{$96 \mathrm{~h}$} \\
& $10 \mathrm{mg} / \mathrm{ml}$ & $40 \mathrm{mg} / \mathrm{ml}$ & $10 \mathrm{mg} / \mathrm{ml}$ & $40 \mathrm{mg} / \mathrm{ml}$ & $10 \mathrm{mg} / \mathrm{ml}$ & $40 \mathrm{mg} / \mathrm{ml}$ \\
\hline SC & $0,14 \pm 0,02^{\mathrm{a}}$ & $0,36 \pm 0,03 \mathrm{a}, \mathrm{d}$ & $0,26 \pm 0,03 \mathrm{a}$ & $0,43 \pm 0,05^{\mathrm{a}}$ & $0,59 \pm 0,02^{\mathrm{a}}$ & $0,52 \pm 0,02^{\mathrm{a}}$ \\
RE & $1,48 \pm 0,32^{\mathrm{b}}$ & $1,97 \pm 0,02^{\mathrm{b}}$ & $0,44 \pm 0,03 \mathrm{~b}$ & $0,54 \pm 0,04^{\mathrm{b}}$ & $0,61 \pm 0,05^{\mathrm{a}}$ & $0,85 \pm 0^{\mathrm{b}}$ \\
PDM & $0,22 \pm 0,01^{\mathrm{a}}$ & $0,16 \pm 0,02 \mathrm{c}$ & $0,14 \pm 0,02 \mathrm{a}$ & $0,16 \pm 0,02^{\mathrm{c}}$ & $0,28 \pm 0,03^{\mathrm{D}}$ & $0,26 \pm 0,04^{\mathrm{c}}$ \\
OZE & $0,39 \pm 0^{\mathrm{a}}$ & $0,42 \pm 0,09 \mathrm{a}$ & $0,54 \pm 0,11 \mathrm{~b}$ & $0,43 \pm 0,02^{\mathrm{a}}$ & $0,52 \pm 0,07^{\mathrm{a}}$ & $0,54 \pm 0,01^{\mathrm{a}}$ \\
Control & $0,26 \pm 0,03^{\mathrm{a}}$ & $0,26 \pm 0,03 \mathrm{c}^{\mathrm{d}}$ & $0,2 \pm 0,03^{\mathrm{a}}$ & $0,2 \pm 0,03^{\mathrm{c}}$ & $0,26 \pm 0,01^{\mathrm{b}}$ & $0,26 \pm 0,01^{\mathrm{c}}$ \\
\hline
\end{tabular}

SC: silicato de calcio, RE: resina epóxica, PDM: poli-dimetilsiloxano, OZE: óxido de zinc eugenol, Control: citotoxicidad normal. Los valores de la misma columna que no comparten el mismo superíndice son significativamente diferentes en $p<.05$.

Tabla III. Resultados de absorbancia obtenidos del análisis de proliferación en osteoblastos exposición al material extraído durante 10 minutos.

\begin{tabular}{lllllll}
\hline \multirow{2}{*}{ Grupos } & \multicolumn{2}{c}{$24 \mathrm{~h}$} & \multicolumn{2}{c}{$72 \mathrm{~h}$} & \multicolumn{2}{c}{$96 \mathrm{~h}$} \\
& $10 \mathrm{mg} / \mathrm{ml}$ & $40 \mathrm{mg} / \mathrm{ml}$ & $10 \mathrm{mg} / \mathrm{ml}$ & $40 \mathrm{mg} / \mathrm{ml}$ & $10 \mathrm{mg} / \mathrm{ml}$ & $40 \mathrm{mg} / \mathrm{ml}$ \\
\hline SC & $0,67 \pm 0,03^{\mathrm{a}}$ & $0,34 \pm 0,02^{\mathrm{a}}$ & $0,49 \pm 0^{\mathrm{a}}$ & $0,36 \pm 0,08 \mathrm{a}$ & $0,19 \pm 0^{\mathrm{a}}$ & $0,71 \pm 0,14^{\mathrm{a}}$ \\
$\mathrm{RE}$ & $0,04 \pm 0,01^{\mathrm{b}}$ & $0 \pm 0^{\mathrm{b}}$ & $0,24 \pm 0,03 \mathrm{~b}$ & $0 \pm 0^{\mathrm{b}}$ & $1,28 \pm 0,02^{\mathrm{b}}$ & $0,99 \pm 0,02^{\mathrm{a}, \mathrm{b}}$ \\
PDM & $0,27 \pm 0,01^{\mathrm{c}}$ & $1,12 \pm 0,2^{\mathrm{c}}$ & $0,58 \pm 0,04 \mathrm{a}$ & $1,75 \pm 0,01 \mathrm{c}$ & $0,66 \pm 0,01^{\mathrm{c}}$ & $0,81 \pm 0,05^{\mathrm{a}}$ \\
OZE & $0,49 \pm 0,06^{\mathrm{d}}$ & $0,15 \pm 0,02^{\mathrm{a}, \mathrm{b}}$ & $0,22 \pm 0,05 \mathrm{~b}$ & $1,98 \pm 0,07 \mathrm{~d}$ & $0,17 \pm 0,02^{\mathrm{a}}$ & $1,33 \pm 0,15^{\mathrm{b}}$ \\
Control $^{\mathrm{b}}$ & $0,34 \pm 0,01^{\mathrm{c}}$ & $0,34 \pm 0,01^{\mathrm{a}}$ & $0,82 \pm 0,06 \mathrm{c}$ & $0,82 \pm 0,06 \mathrm{e}$ & $1,95 \pm 0,2^{\mathrm{d}}$ & $1,95 \pm 0,2^{\mathrm{c}}$ \\
\hline
\end{tabular}

SC: silicato de calcio, RE: resina epóxica, PDM: poli-dimetilsiloxano, OZE: óxido de zinc eugenol, Control: proliferación normal. Los valores de la misma columna que no comparten el mismo superíndice son significativamente diferentes en $p<.05$. 
RAMOS-LÓPEZ, M.; SILVA-BENITEZ, E. L.; AGUILAR-MEDINA, M.; AYALA-HAM, A. R.; ROMERO-QUINTANA, J. G.; SOTO-SAINZ, J. E.; VELÁZQUEZ-SOLANO, A.; RAMOSPAYAN, R. \& CASTRO-SALAZAR, G. Y. Evaluación in vitro de la Biocompatibilidad de Cuatro Cementos Selladores con Osteoblastos Humanos. Int. J. Odontostomat., 13(1):64-68, 2019.

Tabla IV. Resultados de absorbancia obtenidos del análisis de proliferación en osteoblastos exposición al material extraído durante 8 horas.

\begin{tabular}{|c|c|c|c|c|c|c|}
\hline \multirow[b]{2}{*}{ Grupos } & \multicolumn{2}{|c|}{$24 \mathrm{~h}$} & \multicolumn{2}{|c|}{$72 \mathrm{~h}$} & \multicolumn{2}{|c|}{$96 \mathrm{~h}$} \\
\hline & $10 \mathrm{mg} / \mathrm{ml}$ & $40 \mathrm{mg} / \mathrm{ml}$ & $10 \mathrm{mg} / \mathrm{ml}$ & $40 \mathrm{mg} / \mathrm{ml}$ & $10 \mathrm{mg} / \mathrm{ml}$ & $40 \mathrm{mg} / \mathrm{ml}$ \\
\hline SC & $0,42 \pm 0,13^{a}$ & $0,21 \pm 0,01^{a}$ & $0,27 \pm 0,04^{a}$ & $1,21 \pm 0,28^{a}$ & $1,89 \pm 0,07^{\mathrm{a}}$ & $0,12 \pm 0,03 a$ \\
\hline RE & $0,04 \pm 0^{6}$ & $0,01 \pm 0^{b}$ & $0,24 \pm 0,15^{a, c}$ & $0,03 \pm 0,03^{b}$ & $1,11 \pm 0,04^{b}$ & $0,19 \pm 0,01 a, b$ \\
\hline PDM & $0,28 \pm 0,01^{a}$ & $0,31 \pm 0,03^{c}$ & $0,56 \pm 0,05^{b}$ & $0,67 \pm 0,06^{c}$ & $0,79 \pm 0,01^{\mathrm{c}}$ & $0,46 \pm 0,04 b$ \\
\hline OZE & $0,09 \pm 0,01^{b}$ & $0,01 \pm 0^{b}$ & $0,02 \pm 0,01^{c}$ & $1,11 \pm 0,03^{a, d}$ & $1,25 \pm 0,08^{b}$ & $1,71 \pm 0,04 \mathrm{c}$ \\
\hline Control & $0,34 \pm 0,01^{a}$ & $0,34 \pm 0,01^{c}$ & $0,82 \pm 0,06^{d}$ & $0,82 \pm 0,06^{\mathrm{c}, \mathrm{d}}$ & $1,95 \pm 0,2^{a}$ & $1,95 \pm 0,2^{c}$ \\
\hline
\end{tabular}

SC: silicato de calcio, RE: resina epóxica, PDM: poli-dimetilsiloxano, OZE: óxido de zinc eugenol, Control: Proliferación normal. Los valores de la misma columna que no comparten el mismo superíndice son significativamente diferentes en $p<.05$.

\section{DISCUSIÓN}

El objetivo del tratamiento de conductos es regresar al órgano dentario a un estado de salud y funcionalidad sin realizar ningún tipo de intervención quirúrgica (Fernandes \& de Ataide). Para esto el tratamiento debe desarrollarse procurando que los materiales usados para el sellado del conducto permanezcan dentro del conducto radicular.

Los materiales de relleno del canal radicular por si solos no son suficientes para asegurar una exitosa obturación del conducto (Shon et al.), por lo que es necesario utilizar cementos selladores, los cuales pueden ser extruidos o diluidos a través de la constricción apical y de esta forma entrar en contacto con los tejidos perirradiculares (Rodrigues et al.). Por esta razón es importante conocer la biocompatibilidad de los cementos selladores fabricados a base de diferentes materiales para de esta forma tomar la mejor elección clínica. En este estudio se evaluaron cuatro cementos selladores in vitro con diferente composición química: óxido de zinc eugenol (Sealapex; SybroEndo, CA, USA), resina epóxica (AH Plus; Dentsply, Konstanz, Alemania), silicato de calcio (MTA Fillapex; Angelus, PR, Brasil) y polidimetilxilosano (Roeko Seal; Coltene/Whaledent, Langenau, Alemania).

Estos cementos fueron puestos en contacto con medio de cultivo a dos diferentes concentraciones (10 y $40 \mathrm{mg} / \mathrm{ml}$ ) durante dos periodos extracción (10 minutos y $8 \mathrm{~h}$ ), para después ser colocados en contacto con una línea celular primaria humana de osteoblastos durante 24,72 y $96 \mathrm{~h}$.

En relación al análisis de citotoxicidad, los osteoblastos mostraron el mayor porcentaje de muerte celular con el cemento a base de resina epóxica. Pinna et al. evaluaron la citotoxicidad de diferentes cementos durante 5 semanas usando osteoblastos de osteosarcoma de rata, observando que el AH Plus Jet mostró una alta citotoxicidad durante la primera semana, después de la tercera semana la citotoxicidad disminuye siendo comparable con su control, esto concuerda con Loushine et al. que estudiaron AH Plus durante 6 semanas con osteoblastos de ratón. Kim et al. $(2013 a, b)$ también evaluaron la citotoxicidad de AH Plus en una línea tipo osteoblastos de ratón (MC-3T3) mostrando una alta citotoxicidad a las 24 horas.

De la misma manera en un estudio realizado por Xu et al. se evaluó la citotoxicidad de este cemento con un extracto preparado a 72 h de liberación en células tipo osteoblastos (MG63) con diferentes diluciones mostrando una citotoxicidad de moderada a severa en los primeros tres días lo que concuerda con nuestros resultados, estas mismas células fueron evaluadas por Ehsani et al., aunque ellos afirman que son células tipo fibroblastos, mostrando una significativa citotoxicidad a las 24 horas. Garrido et al. evaluaron la viabilidad de células tipo osteoblastos (Osteo-1) mediante azul de tripano colocándolas 24 horas en contacto con extractos preparados a una concentración de $0,1 \mathrm{mg} / \mathrm{ml}$ durante 24 horas, se observó que AH Plus presentó una alta citotoxicidad. Se piensa que esta situación es principalmente debido al componente resinoso y a la amina que promueve su polimerización (Pinna et al.; Xu et al.).

Es importante hacer notar que todos los estudios citados anteriormente (Pinna et al.; Xu et al.; Loushine et al.; Ehsani et al.; Kim et al., 2013a,b; Garrido et al.) evalúan únicamente citotoxicidad, nosotros evaluamos citotoxicidad mediante análisis de LDH y proliferación con MTT. Además, los estudios reportados en osteoblastos hasta el momento no son células humanas, a diferencia de nuestro estudio.

El cemento que mostró la menor citotoxicidad fue el elaborado a base de poli-dimetilxilosano, hasta el momento un solo artículo ha evaluado la biocompatibilidad de este cemento en contacto con una 
RAMOS-LÓPEZ, M.; SILVA-BENITEZ, E. L.; AGUILAR-MEDINA, M.; AYALA-HAM, A. R.; ROMERO-QUINTANA, J. G.; SOTO-SAINZ, J. E.; VELÁZQUEZ-SOLANO, A.; RAMOSPAYAN, R. \& CASTRO-SALAZAR, G. Y. Evaluación in vitro de la Biocompatibilidad de Cuatro Cementos Selladores con Osteoblastos Humanos. Int. J. Odontostomat., 13(1):64-68, 2019.

línea celular de osteoblastos (Al-Awadhi et al.), en este caso de calvaria de rata, mostrando la menor citotoxicidad entre los cementos evaluados por estos autores, lo cual concuerda con nuestros resultados.

Los resultados obtenidos sugieren que el cemento a base de resina epóxica mostró una citotoxicidad marcada, por el contrario, el cemento a base de polidimetilxilosano no presentó citotoxicidad. En relación a la proliferación ninguno de los cementos evaluados mostró resultados alentadores, e inclusive se observó una proliferación muy por debajo de la normal cuando las células fueron expuestas al cemento a base de resina.

El resto de los cementos no mostraron una tendencia específica en relación a la proliferación celular normal.

RAMOS-LÓPEZ, M.; SILVA-BENITEZ, E. L.; AGUILAR-MEDINA, M.; AYALA-HAM, A. R.; ROMERO-QUINTANA, J. G.; SOTO-SAINZ, J. E.; VELÁZQUEZ-SOLANO, A.; RAMOS-PAYAN, R. \& CASTRO-SALAZAR, G. Y. In vitro evaluation of four sealer cements biocompatibility with human osteoblasts. Int. J. Odontostomat., 13(1):69-77, 2019.

ABSTRACT: Correct apical sealing is an important step during root canal treatment, hence, gutta-percha points and sealant are used. There are several chemical compositions on the market, so it is important to evaluate the effects of these in the periapical healing process. The aim of this study was to evaluate the biocompatibility of four sealer cements with different chemical composition placed in contact with human osteoblast. Different extracts were prepared at two concentrations $(10 \mathrm{mg} / \mathrm{mL}$ and 40 $\mathrm{mg} / \mathrm{mL}$ ) and two exposure times (10 min and $8 \mathrm{~h}$ ) these were placed in contact with human osteoblast to evaluate cytotoxicity and proliferation at 24,48 and $72 \mathrm{~h}$ with their respective controls and blanks. A statistical analysis was performed with ANOVA of one factor and Bonferroni post hoc. Results obtained in cytotoxicity and proliferation assays, indicated that the resinbased cement is not biocompatible with osteoblast. The poly-dimethylxilosanbased cement was the only that did not show cytotoxicity at any time of exposure and concentration examined in this study.

KEY WORDS: biocompatibility, sealer cements, osteoblasts.

\section{REFERENCIAS BIBLIOGRÁFICAS}

Al-Awadhi, S.; Spears, R.; Gutmann, J. L. \& Opperman, L.A. Cultured primary osteoblast viability and apoptosis in the presence of root canal sealers. J. Endod., 30(7):527-33, 2004

Bernáth, M. \& Szabó, J. Tissue reaction initiated by different sealers. Int. Endod. J., 36(4):256-61, 2003.

Brackett, M. G.; Messer, R. L.; Lockwood, P. E.; Bryan, T. E.; Lewis, J. B.; Bouillaguet, S. \& Wataha, J. C. Cytotoxic response of three cell lines exposed in vitro to dental endodontic sealers. J. Biomed. Mater. Res. B Appl. Biomater., 95(2):380-6, 2010.

Ehsani, M.; Zabihi, E. \& Gharouee, H. A Comparison between cytotoxicity induced by two resin based sealers (2Seal and AH Plus) in Saos-2 and MG-63 cell lines. Int. J. Mol. Cell Med., 1(4):197-202, 2012.

Eldeniz, A. U.; Mustafa, K.; Ørstavik, D. \& Dahl, J. E. Cytotoxicity of new resin-, calcium hydroxide- and silicone-based root canal sealers on fibroblasts derived from human gingiva and L929 cell lines. Int. Endod. J., 40(5):329-37, 2007

Fernandes, M. \& de Ataide, I. Nonsurgical management of periapical lesions. J. Conserv. Dent., 13(4):240-5, 2010.
Garrido, A. D.; de Cara, S. P.; Marques, M. M.; Sponchiado, E. C. Jr.; Garcia L. da F. \& de Sousa-Neto, M. D. Cytotoxicity evaluation of a copaiba oilbased root canal sealer compared to three commonly used sealers in endodontics. Dent. Res. J. (Isfahan), 12(2):121-6, 2015.

Gerstenfeld, L. C.; Cullinane, D. M.; Barnes, G. L.; Graves, D. T. \& Einhorn, T. A. Fracture healing as a post-natal developmental process: molecular, spatial, and temporal aspects of its regulation. J. Cell. Biochem., 88(5): 873-84, 2003.

Huang, F. M.; Chou, M. Y. \& Chang, Y. C. Induction of cyclooxygenase-2 mRNA and protein expression by epoxy resin and zinc oxide-eugenol based root canal sealers in human osteoblastic cells. Biomaterials, 24(11):1869-75, 2003

Huang, F. M.; Lee, S. S.; Yang, S. F. \& Chang, Y. C. Up-regulation of receptor activator nuclear factor-kappaB ligand expression by root canal sealers in human osteoblastic cells. J. Endod., 35(3):363-6, 2009.

Kim, T. G.; Lee, Y. H.; Bhattari, G.; Lee, N. H.; Lee, K. W.; Yi, H. K. \& Yu, M. K. PPARg inhibits inflammation and RANKL expression in epoxy resinbased sealer-induced osteoblast precursor cells E1 cells. Arch. Oral Biol., 58(1):28-34, 2013a.

Kim, T. G.; Lee, Y. H: Lee, N. H.; Bhattarai, G. Lee, I. K: Yun, B. S. \& Yi, H. $\mathrm{K}$. The antioxidant property of pachymic acid improves bone disturbance against $\mathrm{AH}$ plus-induced inflammation in MC-3T3 E1 cells. J. Endod., 39(4):461-6, 2013b.

Lee, D. H.; Lim, B. S.; Lee, Y. K. \& Yang, H. C. Mechanisms of root canal sealers cytotoxicity on osteoblastic cell line MC3T3-E1. Oral Surg. Oral Med. Oral Pathol. Oral Radiol. Endod., 104(5):717-21, 2007.

Loushine, B. A.; Bryan, T. E.; Looney, S. W.; Gillen, B. M.; Loushine, R. J. Weller, R. N.; Pashley, D. H. \& Tay, F. R. Setting properties and cytotoxicity evaluation of a premixed bioceramic root canal sealer. J. Endod., 37(5):673-7, 2011

Nair, P. N. On the causes of persistent apical periodontitis: a review. Int. Endod. J., 39(4):249-81, 2006.

Pinna, L.; Brackett, M. G.; Lockwood, P. E.; Huffman, B. P.; Mai, S.; Cotti, E.; Dettori, C.; Pashley, D. H. \& Tay, F. R. In vitro cytotoxicity evaluation of a self-adhesive, methacrylate resin-based root canal sealer. J. Endod., 34(9):1085-8, 2008.

Rodrigues, C.; Costa-Rodrigues, J.; Capelas, J. A. \& Fernandes, M. H Behaviour of co-cultured human osteoclastic and osteoblastic cells exposed to endodontic sealers' extracts. Clin. Oral Investig., 18(2):47988, 2014

Salles, L. P.; Gomes-Cornélio, A. L.; Guimarães, F. C.; Herrera, B. S.; Bao, S. N.; Rossa-Junior, C.; Guerreiro-Tanomaru, J. M. \& Tanomaru-Filho, M. Mineral trioxide aggregate-based endodontic sealer stimulates hydroxyapatite nucleation in human osteoblast-like cell culture. J. Endod., 38(7):971-6, 2012

Scelza, M. Z.; Linhares, A. B.; da Silva, L. E.; Granjeiro, J. M. \& Alves, G. G. A multiparametric assay to compare the cytotoxicity of endodontic sealers with primary human osteoblasts. Int. Endod. J., 45(1):12-8, 2012.

Shon, W. J.; Bae, K. S.; Baek, S. H.; Kum, K. Y.; Han, A. R. \& Lee, W. C Effects of calcium phosphate endodontic sealers on the behavior of human periodontal ligament fibroblasts and MG63 osteoblast-like cells. J. Biomed. Mater. Res. B Appl. Biomater., 100(8):2141-7, 2012.

Xu, P.; Liang, J.; Dong, G.; Zheng, L. \& Ye, L. Cytotoxicity of RealSeal on human osteoblast-like MG63 cells. J. Endod., 36(1):40-4, 2010.

Corresponding author:

Castro-Salazar Gloria Yolanda, PhD.

Facultad de Odontalgia

Universidad Autónoma de Sinaloa - Ciudad Universitaria

Josefa Ortiz de Domínguez S/N y Avenida de las Américas CP. 80010. Culiacán, Sinaloa - MÉXICO

Email: yolandacastro@uas.edu.mx.

Recibido : 31-10-2018

Aceptado: $26-11-2018$ 